\title{
Investigating Construction Delay Factors and Their Causes on Reservoir Projects in Vietnam
}

\author{
Dinh Tuan Hai ${ }^{1, *}$, Nguyen Van Son ${ }^{2}$ \\ ${ }^{1}$ Faculty of Civil Engineering, Hanoi Architectural University, Vietnam \\ ${ }^{2}$ Faculty of Civil Engineering, ThuyLoi University, Vietnam
}

Received August 24, 2021; Revised September 29, 2021; Accepted November 11, 2021

\begin{abstract}
Cite This Paper in the following Citation Styles
(a): [1] Dinh Tuan Hai, Nguyen Van Son, "Investigating Construction Delay Factors and Their Causes on Reservoir Projects in Vietnam," Civil Engineering and Architecture, Vol. 9, No. 7, pp. 2338 - 2352, 2021. DOI: 10.13189/cea.2021.090721.
\end{abstract}

(b): Dinh Tuan Hai, Nguyen Van Son (2021). Investigating Construction Delay Factors and Their Causes on Reservoir Projects in Vietnam. Civil Engineering and Architecture, 9(7), 2338 - 2352. DOI: 10.13189/cea.2021.090721.

Copyright@2021 by authors, all rights reserved. Authors agree that this article remains permanently open access under the terms of the Creative Commons Attribution License 4.0 International License

\begin{abstract}
Currently, irrigation and hydropower are important economic and social development sectors that improve the quality and efficiency of people's life in the world as well as Vietnam. Vietnam has constructed around seven thousand reservoirs to date for many purposes such as water supply, flood control, or hydroelectric power. These reservoir construction projects play an important role in using water resources effectively and efficiently. However, many of these projects have been facing construction delays or increasing investment capital. This study aims to investigate the main causes of construction delay of reservoir projects through data collected from 310 valid respondents. Based on comprehensive literature review, this study identified and assessed a total of 37 primary causes which were grouped into six categories, namely, design phrase (six causes), construction phrase (five causes), human factors (five causes), technical factors (10 causes), legal factors (three causes), and external factors (eight causes). The results of regression analysis indicated that causes of technical, external, and human categories were the main causes of construction delays in reservoir projects. In conclusion, this study was useful for both academics and practitioners in exploring the important causes in reservoir construction projects which can help decision-makers reduce construction delays and use resources efficiently.
\end{abstract}

Keywords Causes, Construction, Delay, Projects, Reservoirs, Vietnam

\section{Introduction}

Irrigation and hydropower are important economic and social development sectors that improve the quality and efficiency of Vietnamese people's life. These projects were strengthened by the state during the country's construction and development period. Vietnam currently has approximately 6.648 large and small reservoirs, as well as over 548 hydropower projects [1]. Irrigation and hydropower projects are examples of general outcomes, and they have close working relationships with many people in a variety of fields, including planning, scientific research, surveying, designing, manufacturing, construction, and exploitation management. These projects include many different fields of construction science and technology and have the main following characteristics: (1) huge construction volume with large scale; (2) investment capital is often enormous according to the conditions of each region; (3) contain numerous items, materials, tasks; (4) high-quality requirement; (5) construction conditions difficult; and (6) construction duration is often short.

According to Hue and Son [2], many of these construction projects' schedule is very slow, and many items stop construction annually such as Do Diem gate (Ha Tinh Province), Rao Da reservoir (Quang Binh Province), Nuoc Trong reservoir (Quang Ngai Province), Song Ray reservoir (Ba Ria-Vung Tau Province), Ta Trach reservoir (Hue Province). Some of works which are in preparation stage of project such as Ban Mong (Nghe An Province), upper Krong Pach reservoir (Đak Lak 
Province), LaMo reservoir (Gia Lai Province) have very slow approval process. Examples for 10 cases of project delayed in Vietnam [3] are illustrated as below:

(1) Started in January 2016, project of the repair and upgrading work of Xa Huong reservoir has five large items being implemented: reinforcing dam roof, installing permeability and subsidence monitoring equipment for earth dam system; constructing flood spillway; upgrading water intake; installation of electrical systems and repair and upgrade N2 channel. Currently, all items are being urgently completed by the construction unit, especially the flood spillway item. As a component in the project of repairing and upgrading the Xa Huong reservoir, the flood spillway regulates the water level in the lake, ensures safety for the lake, especially when floods occur. Although, a huge volume is constructed in short time, the flood spillway has basically completed the hydraulic part on 30th April and ensured safety for dam when rainy season occurs.

(2) Ba river in Nghe An Province, which is a main irrigation system, continuous between Yen Thanh and Dien Chau District, and ensures drainage for Phu Thanh Van Thanh, Hop Thanh District, etc. and some of the area in Dien Chau District, have a lot of small branches. Therefore, the dredging of rive is also divided into four routes, including the Choi river route in Van Thanh District, the Choi river in Roc Loi District and regulator in Dien Thai District. Although, predict construction time is 24 months but after five year, the project of repairing and upgrading embankments to prevent erosion and flooding, Dien Yen 2 drainage system of Yen Thanh District has just been completed nearly half. It makes people in this area urgent and worry, especially in the rainy season.

(3) Ban Mong reservoir is main irrigation project of Nghe An Province. Up to this point, a number of main items are constructing ahead of schedule. However, many items have to be shut down due to the lack of construction site.

(4) Son La hydropower is national key project located on Da river in Son La Province. After seven years, Son La hydropower now stands among the thousands of mountains in the Northwest. With a concrete dam the largest height is more than 138m; The total capacity of the reservoir is over 9 billion $\mathrm{m}^{3}$; with a capacity of 2,400 MW with six units, Son La Hydropower has the main task of supplying electricity to the national electricity system with an average annual output of 10,246 billion $\mathrm{kWh}$; competing against floods in the rainy season, supplying water in the dry season for the Northern Delta and contributing to the socio-economic development of the Northwestern region. After seven years of construction, Son La hydropower is the largest national key project in Vietnam and also in Southeast Asia, which has been completed. It also exceeded the three-year schedule and saved 45,000 billion VND of construction cost.

(5) Lai Chau hydropower which has a total investment of 35,700 billion VND, is a special grade construction and is the third largest hydropower in Da river, after Son La and Hoa Binh project, with the main tasks: Supplying electricity to the National Electricity System and serving flood control in the rainy season, supplying water in the dry season for the Northern Delta, and at the same time contributing to promoting socio-economic development of Lai Chau Province. Chau, Dien Bien and the Northwest region, ensuring national defense and security in the Northwest region. Assembly number 2 generates electricity 11 days ahead of schedule, therefore, it generates 60 billion dong. Especially at the current time, when the water level of Lai Chau hydropower reservoir is three meter from the normal rising water level, the average water flow to the lake is $900 \mathrm{~m}^{3} / \mathrm{s}$ and the highest is $1,200 \mathrm{~m}^{3} / \mathrm{s}$. Earlier electricity generating of assembly number 2 will not have to discharge unjustly when the water returns to the lake, and waste water.

(6) Huoi Quang hydropower is invested by Vietnam Electricity Group, built on Nam Mu River, reassure line and reservoir in Than Uyen District, Lai Chau Province. The hydropower is located in Muong La District, Son La Province. Huoi Quang hydropower plant has two assemblies with a total installed capacity of 520MW (2x260), which is the first underground hydropower plant designed and constructed by Vietnam with 2 underground water tunnels in the mountain bed, each tunnel has $4,2 \mathrm{~km}$ length, 7,5m diameter and the generators are also located underground in the mountains. This is one of the major projects in the planning of hydroelectricity on the Da river system, after Son La hydroelectricity (2,400MW), Hoa Binh hydroelectricity (1,920MW) and Lai Chau hydroelectricity $(1,200 \mathrm{MW})$. Assembly number 2 of Huoi Quang hydroelectricity connected to national grid at this time, making a milestone that the project has achieved and aims to complete the whole project in 2016 and responds original approval schedule.

(7) The Ta Trach reservoir project was constructed in late 2005 with a total investment of 3,848 billion VND. At the beginning, the construction revealed many difficulties and challenges due to weather, geology, complicated technical solutions. The main dam, with an embankment volume of 9 million cubic meters, is the most difficult item in the construction process, almost impossible to complete. But since 2010, the weather has been favorable, and the appropriate land treatment plan, the embankment is up to 3 million cubic meters in each year. In October 2016, the project has been completed and filled with water. The reason for delay schedule of embankment dam is due to heavy rain so the soil moisture is very low, not meeting the requirements. According to technical process, before putting on dams, the soil moisture muss be less than or equal to $17 \%$. Meanwhile, the moisture in the land mines is too high compared to the regulations, which makes difficulty in handling humidity and time consumption.

(8) Trung Son Hydropower Plant was started 
construction in November 2012, with a total investment of $\$ 410.68$ million. The plant consists of four assemblies with an installed capacity of $260 \mathrm{MW}$. After nearly four years of construction, up to now, the main items of Trung Son hydropower project such as weir, spillway, and inlet of the plant are on schedule and ready to participate in flood control in 2016.

(9) Cua Dat Reservoir is an irrigation-hydropower project located in the Chu River area (Xuan My Commune), about $17 \mathrm{~km}$ upstream from Bai Thuong dam. With a total investment of nearly 7,000 billion VND, the project includes two parts of irrigation and hydropower with dual-use functions. Cua Dat irrigation works was started in 2004 and was expected to finish construction in May 2009 and stored water for electricity generation during the flood season in 2009. However, until November 27, 2010 the project was completed. The reason is that during the construction process, there was a problem of the flood in 2008, causing the break of the dam under construction at the elevation of $50.0 \mathrm{~m}$ above water level.

(10) As of June 2016, Lao Cai has approved 71 small and medium hydropower projects with a total designed capacity of $1,060.85 \mathrm{MW}$. Up to now, 34 projects (total capacity of $569.8 \mathrm{MW}$ ) have come into operation; 14 projects (total capacity of $247.5 \mathrm{MW}$ ) are delayed in construction; 14 projects (total capacity of $185.35 \mathrm{MW}$ ) have been granted investment certificates; 8 projects (total capacity of 55.2MW) are in the investigation phase; and a $3 \mathrm{MW}$ project has no investor. The reason why many hydropower projects in Lao Cai are delayed is the weak capacity of some investors and construction contractors. Moreover, complicated terrain, abnormal rain and flood make the difficulty in transporting materials, device. Stretching dozens of kilometers of some projects also causes the delay in construction due to site's problem.

It can be seen from general statistics on the implementation of a construction work schedule in general, and hydroelectric and hydraulic works in particular, that the majority of the major irrigation and hydroelectric works were focused from the beginning of construction. It was regularly analyzed to prevent abnormal factors during the process of setting up and managing the construction schedule. As a result, the construction schedule is basically met. Furthermore, small and medium irrigation and hydropower projects are frequently delayed. It is demonstrated that the project management and administration departments play an important role in managing construction progress. In the current climate of climate change and deforestation, these works play a significant role in downstream flood reduction; thus, irrigation and hydropower constructions will be continuously upgraded and repaired in the future. However, there are numerous reasons for the construction delays in Vietnam, including hydrology, technology, engineering, and human factors. The study aims to identify and analyze the causes of reservoir project delays in Vietnam, as well as the relationship between these factors, and then predict these effects by using a regression model.

\section{Literature Reviews}

In the years of World War I, Henry Gantt (1861-1919) proposed the method of making progress in the horizontal chart (bar chart), also known as the Gantt schedule method. This method mainly shows the jobs and time with vertical axis as jobs, horizontal axis is time and each time of job performance is shown in a straight line. By 1930, the slanting schedule method was developed by the United States Army Engineers Association, considered a variant form of the Gantt method. This method has the advantage of expressing the relationship between jobs, the relationship between work and time. By 1956, the network mapping method was created by a combination of Dupont and Remington Rand Univac named CPM (critical path method). Originally built with AOA form (i.e. the job is described as an arrow). Basically, the CPM assumes that during the life of the project only direct costs are lost (ie costs such as materials, machines, labor). The CPM method was introduced to help improve many problems in setting the construction schedule, because it shows the binding relationship between jobs, rather than the use of critical lines in the process of progress management, and construction. However, many scientists realize the limitation of CPM is that the time of construction works depends on the subjective of the schedule maker, while this time depends on many unexpected factors that haven't been counted yet. Therefore, the US Navy introduced the PERT (Program Evaluation and Review Technique) schedule method, which is a variation of CPM. In this method, the work execution time is assumed to be equal to 3 time units: the earliest expected time, the subjective time of the schedule maker and the latest expected time. This method treats the times of a job as a standard distribution probability function. On that basis, conduct calculations according to the theory of statistical probability and give the most optimal project completion time. However, the implicit PERT analysis assumes that all jobs that are not on the critical line are not considered but they are given smaller time values to not alter the original Gang baseline. In actual installation, projects have multiple critical paths instead of a single critical line. Moreover, in random settings, each non-critical work segment can become fully critical and therefore the critical line will be the longest of the set of critical lines. PERT also assumes that time is an independent random variable when in fact it can be completely dependent. Therefore, this method also shows many inaccurate issues.

In Vietnam, there are many studies related to the reasons for delay in construction of projects. Luan [4] 
points out the reasons for delay in construction projects in Vietnam, included ineffective management, weak capacity of contractors, complexity and scale of the project, and limited resources. Lan [5] studies to determine the progress of construction works taking into account the uncertainty in some buildings in Vietnam. Uncertain factors that may delay construction are weather conditions, legal changes, the impact of the economy and the natural environment surrounding the building. Dung [6] in a study aimed to optimize the construction schedule also mentioned the technical and social causes affecting the construction progress. The causes of this construction delay will affect the cost, quality and customer satisfaction at different levels. The statistics of irrigation and hydropower projects 'delays and their main causes in Vietnam is shown on Table 1 [3].

Dam construction, in particular, and construction work in general, have many similarities, so an examination of previous studies is appropriate to serve as the foundation for this research. In many years so far, numerous studies have been focused on causes of delay in construction projects; such as Chan [7], Al-Ghafly [8], Al-Momani [9], Iyer and Jha [10], Sadi and Sadiq [11], Long, et al., [12], El-Razek et al [13], Al-Kharashi and Skitmore [14], Hao and Tam [15], Olawale and Sun [16], Geraldine [17], Anh [18], Lam [19], Ramanathan [20]. As a result, more than 100 factors have the potential to cause construction to be delayed, as provided in Table 2.

Table 1. Statistics of irrigation and hydropower projects 'delays and their main causes in Vietnam

\begin{tabular}{|c|c|c|c|c|}
\hline No & Irrigation and hydropower Projects & $\begin{array}{l}\text { Location in } \\
\text { Vietnam }\end{array}$ & Time Delays & Main Causes \\
\hline 1 & DongTron Reservoir & Phu Yen province & 01 year & $\begin{array}{l}\text { - Unfeasible construction methods } \\
\text { - Late design changes on construction stages }\end{array}$ \\
\hline 2 & T2 Irrigation Canal & Hochiminh city & 04 months & Delays on land clearance progresses \\
\hline 3 & HoaLien Flood Drainage Canal & DaNang city & 06 months & $\begin{array}{c}\text { Overlapping between site surveys, designs and } \\
\text { construction }\end{array}$ \\
\hline 4 & $\begin{array}{c}\text { Irrigation System for North Chu River } \\
\text { - South Ma River }\end{array}$ & ThanhHoa province & 02 years & Unqualified contractors \\
\hline 5 & BaBo Irrigation Canal & Hochiminh city & 06 months & Much heavy rains \\
\hline 6 & KheLai - VucMau Irrigation System & NgheAn province & 06 months & $\begin{array}{l}\text { - Delays on land clearance progresses } \\
\text { - Lack of invested capitals } \\
\text { - Inflation and cost increases } \\
\text { - Changes on material supplied sources }\end{array}$ \\
\hline 7 & CayKhe Reservoir & QuangNgai province & 06 months & Protests from local residents \\
\hline 8 & LocAn Reservoir & DongNai province & 01 year & $\begin{array}{l}\text { - Unusual rainy seasons } \\
\text { - Unsuitable construction methods }\end{array}$ \\
\hline 9 & AngCang Reservoir & DienBien province & 08 years & $\begin{array}{l}\text { - Delays on invested capitals } \\
\text { - Unqualified contractors for solving construction } \\
\text { progress delays } \\
\text { - Unsuitable construction methods } \\
\text { - Inflation and cost increases }\end{array}$ \\
\hline 10 & BacGiang Hydralic Power Station & LangSon province & 06 years & Lack of invested capitals \\
\hline 11 & CamThuy 1 Hydralic Power Station & ThanhHoa province & 05 years & Delays on land clearance progresses \\
\hline 12 & NgoiDuong 1 Hydralic Power Station & LaoCai province & 03 years & Natural disasters and rainy flooding \\
\hline 13 & DakMi 1 Hydralic Power Station & KonTum province & 08 years & $\begin{array}{l}\text { - Lack of invested capitals } \\
\text { - Delays on land clearance progresses } \\
\text { - Unqualified project investors } \\
\end{array}$ \\
\hline 24 & HaDuc 2 Pumping Station & Hanoi city & 03 years & Unsuitable design solutions \\
\hline 25 & PhuMy và DucPho Pumping Station & LamDong province & 01 year & Delays on land clearance progresses \\
\hline 26 & YenNghia Pumping Station & HaNoi province & 06 months & $\begin{array}{l}\text { - Sand and serious underwater flowing } \\
\text { - Delays on land clearance progresses }\end{array}$ \\
\hline 33 & ThanhLam - PhuDa Irigation System & $\begin{array}{l}\text { ThuaThien-Hue } \\
\text { province }\end{array}$ & 09 months & $\begin{array}{l}\text { - Site difficulties for construction } \\
\text { - Lack of invested capitals }\end{array}$ \\
\hline 35 & NongCong Drainage System & ThanhHoa province & 06 months & Delays on land clearance progresses \\
\hline
\end{tabular}


Table 2. Summary of factors from other passed construction projects

\begin{tabular}{|c|c|c|c|c|c|c|c|c|c|c|c|c|c|c|c|}
\hline & & & & & eratı & e sum & tary 0 & factor & from & ther p & ssed c & nstru & ion pr & jects & \\
\hline No. & Subject & Causes of delay & [7] & [8] & [9] & [10] & [11] & [12] & [13] & [14] & [15] & [16] & [17] & [18] & [19] \\
\hline 1 & Contract & $\begin{array}{c}\text { Original contract } \\
\text { duration is too short }\end{array}$ & & & & $\mathrm{x}$ & & & & & & $\mathrm{x}$ & $\mathrm{x}$ & $\mathrm{x}$ & \\
\hline 2 & Contract & $\begin{array}{c}\text { Legal disputes } \\
\text { between parties in } \\
\text { the contract }\end{array}$ & & & & & $\mathrm{x}$ & $\mathrm{x}$ & $\mathrm{x}$ & & $\mathrm{x}$ & & $\mathrm{x}$ & & \\
\hline 3 & Contract & $\begin{array}{c}\text { Inadequate definition } \\
\text { of substile } \\
\text { completion }\end{array}$ & & & & & & & & & & & $\mathrm{x}$ & & $\mathrm{x}$ \\
\hline 4 & Contract & $\begin{array}{c}\text { Ineffective delay } \\
\text { penalties }\end{array}$ & & & & & & & & & & & $\mathrm{x}$ & & $\mathrm{x}$ \\
\hline 5 & Contract & $\begin{array}{c}\text { Type of construction } \\
\text { contract }\end{array}$ & & & & & & & & & & & $\mathrm{x}$ & & \\
\hline 6 & Contract & $\begin{array}{c}\text { Types of project } \\
\text { bidding and awards }\end{array}$ & & & & & & & & & & & $\mathrm{x}$ & $\mathrm{x}$ & \\
\hline 7 & Owner & Investment capital & & & $\mathrm{x}$ & & & $\mathrm{x}$ & & & & & & $\mathrm{x}$ & $\mathrm{x}$ \\
\hline 8 & Owner & $\begin{array}{c}\text { Employees of } \\
\text { owners have limited } \\
\text { capacity }\end{array}$ & & & & & & $\mathrm{x}$ & & $\mathrm{x}$ & & & $\mathrm{x}$ & $\mathrm{x}$ & $\mathrm{x}$ \\
\hline 9 & Owner & $\begin{array}{l}\text { Delay to deliver the } \\
\text { site to the contract }\end{array}$ & & & & & & & & & & $\mathrm{x}$ & $\mathrm{x}$ & & $\mathrm{x}$ \\
\hline 10 & Owner & $\begin{array}{l}\text { High quality } \\
\text { requirement }\end{array}$ & $\mathrm{x}$ & & & & & & $\mathrm{x}$ & $\mathrm{x}$ & $\mathrm{x}$ & & $\mathrm{x}$ & & \\
\hline 11 & Owner & Delay in payment & $\mathrm{x}$ & & & & $\mathrm{x}$ & $\mathrm{x}$ & $\mathrm{x}$ & & $\mathrm{x}$ & $\mathrm{x}$ & $\mathrm{x}$ & & \\
\hline 12 & Owner & $\begin{array}{l}\text { Delay in proving } \\
\text { design documents }\end{array}$ & & & & & & & & & & $\mathrm{x}$ & $\mathrm{x}$ & & $\mathrm{x}$ \\
\hline 13 & Owner & $\begin{array}{l}\text { Changes of initial } \\
\text { investment tasks }\end{array}$ & & & $\mathrm{x}$ & & $\mathrm{x}$ & $\mathrm{x}$ & $\mathrm{x}$ & $\mathrm{x}$ & $\mathrm{x}$ & $\mathrm{x}$ & $\mathrm{x}$ & $\mathrm{x}$ & \\
\hline 14 & Owner & $\begin{array}{c}\text { Delay to revising and } \\
\text { approving design } \\
\text { documents } \\
\end{array}$ & & & & & & & & & & $\mathrm{x}$ & $\mathrm{x}$ & & $\mathrm{x}$ \\
\hline 15 & Owner & $\begin{array}{c}\text { Delay in approving } \\
\text { shop drawings and } \\
\text { sample materials }\end{array}$ & & & & & & & & & & $\mathrm{x}$ & $\mathrm{x}$ & & \\
\hline 16 & Owner & $\begin{array}{l}\text { Poor communication } \\
\text { and coordination by } \\
\text { owner and others }\end{array}$ & & & & & & & & & $\mathrm{x}$ & & $\mathrm{x}$ & $\mathrm{x}$ & $\mathrm{x}$ \\
\hline 17 & Owner & $\begin{array}{c}\text { Slowness in decision } \\
\text { making process }\end{array}$ & & & & & & & & & & $\mathrm{x}$ & $\mathrm{x}$ & $\mathrm{x}$ & $\mathrm{x}$ \\
\hline 18 & Owner & $\begin{array}{c}\text { Conflicts between } \\
\text { joint-ownership of } \\
\text { the project }\end{array}$ & & & & & & & & & $\mathrm{x}$ & & $\mathrm{x}$ & & \\
\hline 19 & Owner & $\begin{array}{l}\text { Unavailability of } \\
\text { incentives for } \\
\text { contractor for } \\
\text { finishing ahead } \\
\text { schedule }\end{array}$ & & & & & & & & & & & $\mathrm{x}$ & $\mathrm{x}$ & $\mathrm{x}$ \\
\hline 20 & Owner & $\begin{array}{l}\text { Suspension of work } \\
\text { by owner }\end{array}$ & & & & & & & & & & & $\mathrm{x}$ & & \\
\hline 21 & Owner & $\begin{array}{c}\text { Legal on patent } \\
\text { issues }\end{array}$ & & $\mathrm{x}$ & & $\mathrm{x}$ & & $\mathrm{x}$ & & & & & & & \\
\hline 22 & Owner & $\begin{array}{c}\text { Shortage of project } \\
\text { budget }\end{array}$ & & $\mathrm{x}$ & $\mathrm{x}$ & & $\mathrm{x}$ & $\mathrm{x}$ & & $\mathrm{x}$ & & $\mathrm{x}$ & & $\mathrm{x}$ & $\mathrm{x}$ \\
\hline 23 & Owner & $\begin{array}{l}\text { Progress is too short } \\
\text { and cannot be } \\
\text { changed }\end{array}$ & & $\mathrm{x}$ & & & $\mathrm{x}$ & & $\mathrm{x}$ & & $\mathrm{x}$ & & & & \\
\hline 24 & Owner & $\begin{array}{l}\text { Signing too many } \\
\text { contracts and } \\
\text { sub-contracting } \\
\text { contracts }\end{array}$ & & & & & & & & & & & $\mathrm{x}$ & $\mathrm{x}$ & $\mathrm{x}$ \\
\hline 25 & Owner & $\begin{array}{l}\text { Rework due to errors } \\
\text { during construction }\end{array}$ & & & & & & & & & & $\mathrm{x}$ & $\mathrm{x}$ & & $\mathrm{x}$ \\
\hline
\end{tabular}


Table 2 Continued

\begin{tabular}{|c|c|c|c|c|c|c|c|c|c|c|c|c|c|c|c|}
\hline 26 & Owner & $\begin{array}{l}\text { Late payment of } \\
\text { main or general } \\
\text { contractors to } \\
\text { sub-contractor }\end{array}$ & $\mathrm{x}$ & & & & $x$ & & & & & $\mathrm{x}$ & $\mathrm{x}$ & $\mathrm{x}$ & \\
\hline 27 & Owner & $\begin{array}{c}\text { Poor supervision and } \\
\text { management }\end{array}$ & & & & & & & & & & & $\mathrm{x}$ & $\mathrm{x}$ & $\mathrm{x}$ \\
\hline 28 & Owner & $\begin{array}{c}\text { Conflicts between } \\
\text { contractor and others }\end{array}$ & & $\mathrm{x}$ & $\mathrm{x}$ & & $\mathrm{x}$ & $\mathrm{x}$ & $\mathrm{x}$ & $\mathrm{x}$ & $\mathrm{x}$ & & $\mathrm{x}$ & $\mathrm{x}$ & $\mathrm{x}$ \\
\hline 29 & Owner & $\begin{array}{c}\text { Unreasonable } \\
\text { construction methods }\end{array}$ & & $\mathrm{x}$ & & & $\mathrm{x}$ & & $\mathrm{x}$ & & & & & $\mathrm{x}$ & $\mathrm{x}$ \\
\hline 30 & Owner & $\begin{array}{l}\text { Change orders by } \\
\text { owner }\end{array}$ & & $\mathrm{x}$ & & & & & & & & & & & $\mathrm{x}$ \\
\hline 31 & Owner & $\begin{array}{c}\text { Unreasonable } \\
\text { construction } \\
\text { schedule } \\
\end{array}$ & $\mathrm{x}$ & & $\mathrm{x}$ & & $x$ & $\mathrm{x}$ & & $\mathrm{x}$ & & & & $\mathrm{x}$ & $\mathrm{x}$ \\
\hline 32 & Owner & $\begin{array}{c}\text { Contractors' job } \\
\text { statistics are } \\
\text { incomplete } \\
\end{array}$ & & & & & & & & & & & $\mathrm{x}$ & & \\
\hline 33 & Owner & $\begin{array}{l}\text { Regular change of } \\
\text { sub-contractors }\end{array}$ & & & & & & & & & & $\mathrm{x}$ & $\mathrm{x}$ & & \\
\hline 34 & Owner & $\begin{array}{c}\text { Poor qualification of } \\
\text { the sub-contractor's } \\
\text { technical staff }\end{array}$ & & & & & & & & & & & $\mathrm{x}$ & & $\mathrm{x}$ \\
\hline 35 & Owner & $\begin{array}{c}\text { Delay in equipment } \\
\text { mobilization }\end{array}$ & & & $\mathrm{x}$ & & & & & & & & $\mathrm{x}$ & & $\mathrm{x}$ \\
\hline 36 & Owner & $\begin{array}{l}\text { Delay in performing } \\
\text { inspection and } \\
\text { testing by consultant }\end{array}$ & & & & & & & & & & $\mathrm{x}$ & $\mathrm{x}$ & & $\mathrm{x}$ \\
\hline 37 & Owner & $\begin{array}{l}\text { Delay approval for } \\
\text { major changes in the } \\
\text { scope of work by } \\
\text { consultants }\end{array}$ & & & & & & & & & & & $\mathrm{x}$ & & $\mathrm{x}$ \\
\hline 38 & Owner & $\begin{array}{c}\text { Inflexibility of } \\
\text { consultant }\end{array}$ & & & & & & & $\mathrm{x}$ & & & $\mathrm{x}$ & $\mathrm{x}$ & & $\mathrm{x}$ \\
\hline 39 & Owner & $\begin{array}{c}\text { Poor communication/ } \\
\text { coordination between } \\
\text { consultant and other } \\
\text { parties }\end{array}$ & & & & & & & & & & & $x$ & $x$ & $\mathrm{x}$ \\
\hline 40 & Owner & $\begin{array}{l}\text { Late in reviewing and } \\
\text { approving design } \\
\text { documents by } \\
\text { consultants }\end{array}$ & & & & & & & & & & & $\mathrm{x}$ & & $\mathrm{x}$ \\
\hline 41 & Owner & $\begin{array}{l}\text { Conflicts between } \\
\text { consultants and } \\
\text { design engineers }\end{array}$ & & & & & & & & & & $\mathrm{x}$ & $\mathrm{x}$ & $\mathrm{x}$ & $\mathrm{x}$ \\
\hline 42 & Owner & $\begin{array}{c}\text { Inadequate } \\
\text { experience of } \\
\text { consultant }\end{array}$ & & & & & & & & & & $\mathrm{x}$ & $\mathrm{x}$ & $\mathrm{x}$ & $\mathrm{x}$ \\
\hline 43 & Owner & $\begin{array}{c}\text { Construction } \\
\text { management is less } \\
\text { safe and effective }\end{array}$ & & & & $\mathrm{x}$ & $\mathrm{x}$ & & & & & $\mathrm{x}$ & & & \\
\hline 44 & Owner & $\begin{array}{c}\text { Lack of safety } \\
\text { insurance }\end{array}$ & & & & $\mathrm{x}$ & & $\mathrm{x}$ & $\mathrm{x}$ & & & $\mathrm{x}$ & & & \\
\hline 45 & Owner & $\begin{array}{c}\text { Pollution related to } \\
\text { construction } \\
\text { activities }\end{array}$ & & & & & & & $\mathrm{x}$ & & & & & & \\
\hline 46 & Owner & $\begin{array}{c}\text { Change in } \\
\text { construction } \\
\text { materials without } \\
\text { approval } \\
\end{array}$ & & & & & & $\mathrm{x}$ & & & & & & & \\
\hline 47 & Owner & $\begin{array}{l}\text { The sub-contractor } \\
\text { complies poorly with } \\
\text { the signed contract }\end{array}$ & $x$ & & & & & $\mathrm{x}$ & & $x$ & & & & & $\mathrm{x}$ \\
\hline
\end{tabular}


Table 2 Continued

\begin{tabular}{|c|c|c|c|c|c|c|c|c|c|c|c|c|c|c|c|}
\hline 48 & Owner & $\begin{array}{c}\text { Sub-contractors have } \\
\text { poor management } \\
\text { capacity } \\
\end{array}$ & & $\mathrm{x}$ & & $\mathrm{x}$ & $\mathrm{x}$ & $\mathrm{x}$ & & $\mathrm{x}$ & & & & & \\
\hline 49 & Owner & $\begin{array}{l}\text { Delay in supplying } \\
\text { materials due to } \\
\text { sub-contractor's } \\
\text { error }\end{array}$ & $\mathrm{x}$ & & & & & & $\mathrm{x}$ & $\mathrm{x}$ & & $\mathrm{x}$ & & & \\
\hline 50 & Owner & $\begin{array}{l}\text { Imperfect conditions } \\
\text { for construction }\end{array}$ & & & & & $\mathrm{x}$ & $\mathrm{x}$ & & & & & & & \\
\hline 51 & Owner & $\begin{array}{c}\text { Un-use of advanced } \\
\text { technology }\end{array}$ & $\mathrm{x}$ & & & & & $\mathrm{x}$ & $\mathrm{x}$ & & & $\mathrm{x}$ & & & \\
\hline 52 & Owner & $\begin{array}{l}\text { Prestige and } \\
\text { experience of } \\
\text { contractors }\end{array}$ & & & & & $\mathrm{x}$ & & & & & $\mathrm{x}$ & & & \\
\hline 53 & Owner & $\begin{array}{c}\text { Poor labor } \\
\text { management }\end{array}$ & $\mathrm{x}$ & & & & $\mathrm{x}$ & & & & & & & & \\
\hline 54 & Owner & $\begin{array}{c}\text { Lack of professional } \\
\text { staff and qualified } \\
\text { managers } \\
\end{array}$ & & $\mathrm{x}$ & $\mathrm{x}$ & & $\mathrm{x}$ & & $\mathrm{x}$ & $\mathrm{x}$ & & $\mathrm{x}$ & & $\mathrm{x}$ & \\
\hline 55 & Owner & $\begin{array}{l}\text { Conflicts between } \\
\text { labor and manager }\end{array}$ & & & $\mathrm{x}$ & & $\mathrm{x}$ & $\mathrm{x}$ & & & & $\mathrm{x}$ & & & \\
\hline 56 & Design & $\begin{array}{c}\text { Mistakes and } \\
\text { discrepancies in } \\
\text { design documents }\end{array}$ & $\mathrm{x}$ & $\mathrm{x}$ & & & $\mathrm{x}$ & $\mathrm{x}$ & $\mathrm{x}$ & $\mathrm{x}$ & $\mathrm{x}$ & & $\mathrm{x}$ & $\mathrm{x}$ & $\mathrm{x}$ \\
\hline 57 & Design & $\begin{array}{c}\text { Delays in producing } \\
\text { design documents }\end{array}$ & & & & & & & & & & & $\mathrm{x}$ & & \\
\hline 58 & Design & $\begin{array}{c}\text { Unclear and } \\
\text { inadequate details in } \\
\text { drawings }\end{array}$ & $\mathrm{x}$ & $\mathrm{x}$ & & $\mathrm{x}$ & & $\mathrm{x}$ & $\mathrm{x}$ & $\mathrm{x}$ & & $\mathrm{x}$ & $\mathrm{x}$ & & $\mathrm{x}$ \\
\hline 59 & Design & $\begin{array}{l}\text { Complexity of } \\
\text { project design }\end{array}$ & & & & & & & & & & & $\mathrm{x}$ & & \\
\hline 60 & Design & $\begin{array}{c}\text { Insufficient data } \\
\text { collection and survey } \\
\text { before design }\end{array}$ & & & & & $\mathrm{x}$ & $\mathrm{x}$ & & & $\mathrm{x}$ & & $\mathrm{x}$ & $\mathrm{x}$ & $\mathrm{x}$ \\
\hline 61 & Design & $\begin{array}{l}\text { Misunderstanding of } \\
\text { owner's } \\
\text { requirements by } \\
\text { design engineer }\end{array}$ & & & & & & & & & & & $\mathrm{x}$ & & \\
\hline 62 & Design & $\begin{array}{l}\text { Inadequate design - } \\
\text { team experience }\end{array}$ & & & & & & & & & & & $\mathrm{x}$ & & \\
\hline 63 & Design & $\begin{array}{c}\text { Un-use of advanced } \\
\text { engineering design } \\
\text { software } \\
\end{array}$ & & & & & & & & & & & $\mathrm{x}$ & & \\
\hline 64 & Design & $\begin{array}{c}\text { Change design } \\
\text { during construction }\end{array}$ & & $\mathrm{x}$ & $\mathrm{x}$ & & $\mathrm{x}$ & & $\mathrm{x}$ & $\mathrm{x}$ & $\mathrm{x}$ & $\mathrm{x}$ & & & $\mathrm{x}$ \\
\hline 65 & Material & $\begin{array}{c}\text { Shortage of } \\
\text { construction } \\
\text { materials in market }\end{array}$ & & & & & & & & & & & $\mathrm{x}$ & & $\mathrm{x}$ \\
\hline 66 & Material & $\begin{array}{l}\text { Changes in material } \\
\text { types and } \\
\text { specifications during } \\
\text { construction }\end{array}$ & & & & & & & & & & & $\mathrm{x}$ & & \\
\hline 67 & Material & $\begin{array}{l}\text { Delay in material } \\
\text { delivery }\end{array}$ & & & & & & & & & & $\mathrm{x}$ & $\mathrm{x}$ & & $x$ \\
\hline 68 & Material & $\begin{array}{l}\text { Damage of sorted } \\
\text { material while they } \\
\text { are needed urgently }\end{array}$ & & & & & & & & & & & $\mathrm{x}$ & & \\
\hline 69 & Material & $\begin{array}{l}\text { Delay in manufacture } \\
\text { for special materials }\end{array}$ & & & & & & & & & & & $\mathrm{x}$ & & \\
\hline 70 & Material & $\begin{array}{l}\text { Late material } \\
\text { procurements }\end{array}$ & & & & & & & & & & & $\mathrm{x}$ & & \\
\hline
\end{tabular}


Table 2 Continued

\begin{tabular}{|c|c|c|c|c|c|c|c|c|c|c|c|c|c|c|c|}
\hline 71 & Material & $\begin{array}{l}\text { Late in selection of } \\
\text { finishing materials } \\
\text { due to availability of } \\
\text { many types in market }\end{array}$ & & $\mathrm{x}$ & & & $\mathrm{x}$ & $\mathrm{x}$ & $\mathrm{x}$ & & & & $\mathrm{x}$ & & \\
\hline 72 & Equipment & $\begin{array}{c}\text { Equipment } \\
\text { breakdowns }\end{array}$ & & & & & & & & & & & $\mathrm{x}$ & & \\
\hline 73 & Equipment & $\begin{array}{c}\text { Shortage of } \\
\text { equipment }\end{array}$ & & & & & & & & & & & $\mathrm{x}$ & $\mathrm{x}$ & \\
\hline 74 & Equipment & $\begin{array}{c}\text { Low level of } \\
\text { equipment-operator's } \\
\text { skill } \\
\end{array}$ & & & & & & & & & & & $\mathrm{x}$ & & \\
\hline 75 & Equipment & $\begin{array}{l}\text { Low productivity and } \\
\text { efficiency of } \\
\text { equipment }\end{array}$ & & & & & $\mathrm{x}$ & $\mathrm{x}$ & & & & $\mathrm{x}$ & $\mathrm{x}$ & & \\
\hline 76 & Equipment & $\begin{array}{c}\text { Lack in } \\
\text { high-technology } \\
\text { mechanical } \\
\text { equipment } \\
\end{array}$ & & & & & & & & & & & $\mathrm{x}$ & & \\
\hline 77 & Labor & Shortage of labors & & & & & & & & & & & $\mathrm{x}$ & & \\
\hline 78 & Labor & $\begin{array}{l}\text { Unqualified } \\
\text { workforce }\end{array}$ & & $\mathrm{x}$ & $\mathrm{x}$ & & & $\mathrm{x}$ & $\mathrm{x}$ & $\mathrm{x}$ & & $\mathrm{x}$ & $\mathrm{x}$ & $\mathrm{x}$ & \\
\hline 79 & Labor & Nationality of labors & & & & & & & & & & & $\mathrm{x}$ & & \\
\hline 80 & Labor & $\begin{array}{l}\text { Low productivity } \\
\text { level of labors }\end{array}$ & & $\mathrm{x}$ & & & $\mathrm{x}$ & & & & & $\mathrm{x}$ & $\mathrm{x}$ & & \\
\hline 81 & Labor & $\begin{array}{c}\text { Personal conflicts } \\
\text { among labors }\end{array}$ & & & & & & & & & & & $\mathrm{x}$ & & \\
\hline 82 & External & $\begin{array}{l}\text { Effects of subsurface } \\
\text { conditions (e.g., soil, } \\
\text { high water table, etc.) }\end{array}$ & $\mathrm{x}$ & $\mathrm{x}$ & $\mathrm{x}$ & $\mathrm{x}$ & $\mathrm{x}$ & $\mathrm{x}$ & $\mathrm{x}$ & $\mathrm{x}$ & & $\mathrm{x}$ & $\mathrm{x}$ & $\mathrm{x}$ & \\
\hline 83 & External & $\begin{array}{c}\text { Delay in obtaining } \\
\text { permits from } \\
\text { municipality }\end{array}$ & & & & & $\mathrm{x}$ & & $\mathrm{x}$ & & $\mathrm{x}$ & & $\mathrm{x}$ & & \\
\hline 84 & External & Hot weather & $\mathrm{x}$ & $\mathrm{x}$ & $\mathrm{x}$ & $\mathrm{x}$ & $\mathrm{x}$ & $\mathrm{x}$ & $\mathrm{x}$ & $\mathrm{x}$ & & $\mathrm{x}$ & $\mathrm{x}$ & & $\mathrm{x}$ \\
\hline 85 & External & $\begin{array}{c}\text { Rain effect on } \\
\text { construction } \\
\text { activities } \\
\end{array}$ & $\mathrm{x}$ & $\mathrm{x}$ & $\mathrm{x}$ & $\mathrm{x}$ & $\mathrm{x}$ & $\mathrm{x}$ & $\mathrm{x}$ & $\mathrm{x}$ & & $\mathrm{x}$ & $\mathrm{x}$ & & $\mathrm{x}$ \\
\hline 86 & External & $\begin{array}{c}\text { Unavailability of } \\
\text { utilities in site (such } \\
\text { as water, electricity, } \\
\text { telephone, etc.) }\end{array}$ & & & & & & & & & & & $\mathrm{x}$ & & \\
\hline 87 & External & $\begin{array}{c}\text { Social and cultural } \\
\text { factors }\end{array}$ & & & & & & & & & & & $\mathrm{x}$ & & \\
\hline 88 & External & $\begin{array}{c}\text { Traffic control and } \\
\text { restriction at job site }\end{array}$ & & & & & & & & & & & $\mathrm{x}$ & & \\
\hline 89 & External & $\begin{array}{c}\text { Accident during } \\
\text { construction }\end{array}$ & & & & $\mathrm{x}$ & $\mathrm{x}$ & $\mathrm{x}$ & $\mathrm{x}$ & & $\mathrm{x}$ & $\mathrm{x}$ & $\mathrm{x}$ & & \\
\hline 90 & External & $\begin{array}{c}\text { Differing site } \\
\text { conditions }\end{array}$ & & & & & & & & & & $\mathrm{x}$ & $\mathrm{x}$ & $\mathrm{x}$ & \\
\hline 91 & External & $\begin{array}{c}\text { Changes in } \\
\text { government } \\
\text { regulations and laws }\end{array}$ & & & & & & & & & & & $\mathrm{x}$ & & \\
\hline 92 & External & $\begin{array}{l}\text { Delay in providing } \\
\text { services from utilities } \\
\text { (such as water, } \\
\text { electricity) }\end{array}$ & & & & & & & & & & & $\mathrm{x}$ & & \\
\hline 93 & External & $\begin{array}{c}\text { Delay in final } \\
\text { inspection and } \\
\text { certification by third } \\
\text { parties } \\
\end{array}$ & & & & & & & & & & & $\mathrm{x}$ & & \\
\hline 94 & External & Inflationary & $\mathrm{x}$ & & $\mathrm{x}$ & & $\mathrm{x}$ & $\mathrm{x}$ & & $\mathrm{x}$ & $\mathrm{x}$ & & $\mathrm{x}$ & $\mathrm{x}$ & \\
\hline 95 & External & $\begin{array}{c}\text { Increase lending } \\
\text { rates }\end{array}$ & $\mathrm{x}$ & & $\mathrm{x}$ & & & $\mathrm{x}$ & & $\mathrm{x}$ & $\mathrm{x}$ & & $\mathrm{x}$ & & \\
\hline
\end{tabular}


Table 2 Continued

\begin{tabular}{|c|c|c|c|c|c|c|c|c|c|c|c|c|}
\hline 96 & External & $\begin{array}{c}\text { Material costs } \\
\text { suddenly increased }\end{array}$ & & $\mathrm{x}$ & $\mathrm{x}$ & $x$ & & $\mathrm{x}$ & $\mathrm{x}$ & $\mathrm{x}$ & $\mathrm{x}$ & \\
\hline 97 & External & $\begin{array}{l}\text { Government } \\
\text { bureaucracy }\end{array}$ & $\mathrm{x}$ & $x$ & & $\mathrm{x}$ & & & $\mathrm{x}$ & & & $x$ \\
\hline 98 & External & $\begin{array}{l}\text { The government's } \\
\text { inappropriate } \\
\text { decision }\end{array}$ & $x$ & $x$ & $\mathrm{x}$ & & & & & & $\mathrm{x}$ & \\
\hline 99 & External & $\begin{array}{c}\text { Formal approval } \\
\text { procedures are } \\
\text { complicated } \\
\end{array}$ & & $\mathrm{x}$ & $\mathrm{x}$ & & $\mathrm{x}$ & & $\mathrm{x}$ & & $\mathrm{x}$ & $\mathrm{x}$ \\
\hline 100 & External & $\begin{array}{c}\text { Unstable government } \\
\text { policy }\end{array}$ & $\mathrm{x}$ & $\mathrm{x}$ & $\mathrm{x}$ & & $\mathrm{x}$ & & $\mathrm{x}$ & & $\mathrm{x}$ & $x$ \\
\hline
\end{tabular}

\section{Research Methodology}

To achieve the goal, this study adopted both qualitative and quantitative approaches to identify and assess the causes of construction delays of irrigation and hydropower projects.

\subsection{Findings from Qualitative Method}

After synthesizing and analyzing previous researches, and exchanging with experts in the field of irrigation and hydropower, delay factors are reduced to 32 main delay causes and assembled into six groups: design factors, external factors, process factors, legal factors, human factors and technical factors. This is a difference because construction delay causes from previous studies are grouped by all project parties involved (owners, contractors, designers, supervisors, etc.); this category has overlaps by mutual impact of each participant. Research model is suggested in Figure 1.

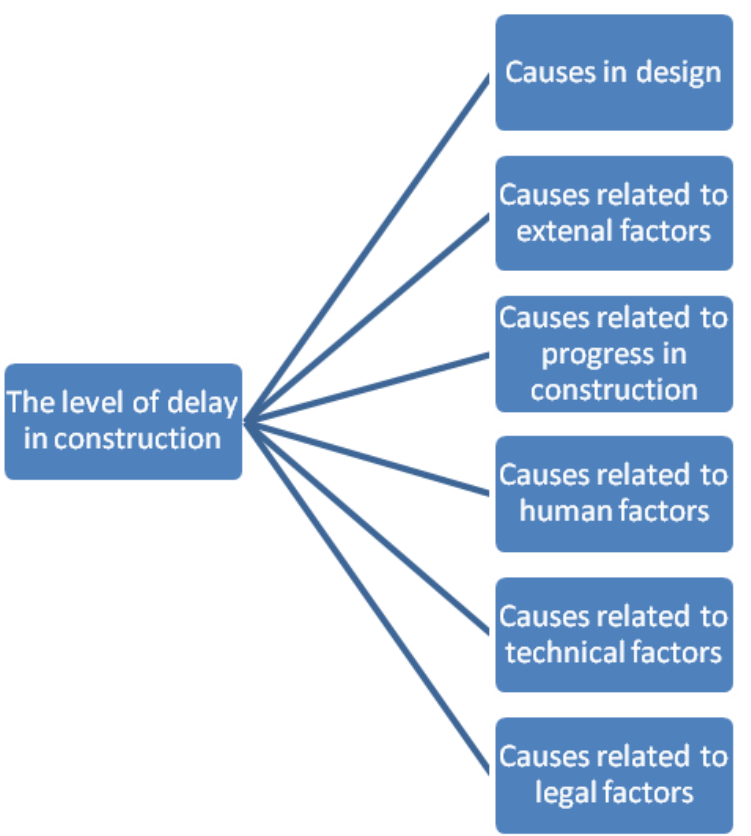

Figure 1. Research Model

\subsection{Questionnaires}

The questionnaire includes six groups with five design factors, six external factors, five progress factors, five human factors, three legal factors and eight technical factors with Influence and Frequency Degrees of Factors as shown on Table 3. Highest influence degree caused delays for construction projects is denoted as "Very High" while "Very Low" means less influence on construction delays. Meantime, "Rarely" factor has less occurrence on construction projects and most occurrence factor is denoted for "Very Often".

Table 3. Influence and Frequency Degrees of Factors

\begin{tabular}{|c|c|c|c|}
\hline \multicolumn{2}{|c|}{ Influence Degrees of Factors } & \multicolumn{2}{c|}{ Frequency Degree of Factors } \\
\hline Level & $\begin{array}{c}\text { Influence } \\
\text { Degrees }\end{array}$ & Level & Frequency Degrees \\
\hline 1 & Very Low & 1 & Rarely \\
\hline 2 & Low & 2 & Sometimes \\
\hline 3 & Moderate & 3 & Moderate \\
\hline 4 & High & 4 & Often \\
\hline 5 & Very High & 5 & Very Often \\
\hline
\end{tabular}

\subsection{Information of Respondents}

Respondents are owners, individual in construction units of irrigation and hydropower projects, individuals of design consultancy units; consulting and supervising irrigation and hydropower projects. They are grouped by number of experience year: less than 3 years, from 3 to 5 years, from 5 to 10 years, from 10 to 15 years and above 15 years. The questionnaires are delivered by post and collected via the google form.

\section{Data Analysis}

\subsection{Information of Research Samples}

There are 310 valid answer sheets after collecting survey results. In particular, the responses were collected from 35/64 provinces in Vietnam. About experience year, $15.8 \%$ of respondents have less than 3 years 'experience 
and $11.3 \%$ have 3 to 5 years, $33.2 \%$ have 5 to 10 years, $25.2 \%$ have 10 to 15 years, $14.5 \%$ have more than 15 years of experience. About the participants of respondents, State owners have $39.4 \%$, project management consultants have $4.9 \%$, supervisor consultants have $6.5 \%$, contractors have $3.2 \%$ and design consultants have $16.2 \%$.

\subsection{Factor Analysis}

After collecting answer sheet, step 1 is conducted: Preliminary examination of the reliability of the scale with Cronbach Alpha coefficient. This method helps eliminate unsuitable variables for component scale through fail corrected item-total correlation. Step 2: After evaluating all the scales through Cronbach Alpha coefficients, Exploratory Factor Analysis-EPA is used, in which KMO (Kaiser-Meyer-Olkin) is a consumer index to consider the appropriateness of EFA with the conditions $\mathrm{KMO} \geq 0.5$. After the observed variables included in EFA, factors reduce to six groups and 32 observed variables as shown on Table 4,5,6,7,8,9 as:

Table 4. Factor 1 - Design Factors

\begin{tabular}{|c|c|}
\hline TK2 & Unclear and inadequate details in drawings \\
\hline TK4 & Change the design during construction time \\
\hline TK6 & $\begin{array}{c}\text { Misunderstanding of owner's requirements by design } \\
\text { engineer }\end{array}$ \\
\hline TK3 & Insufficient data collection and survey before design \\
\hline TK1 & $\begin{array}{c}\text { Increase the work compared to the original investment } \\
\text { policy }\end{array}$ \\
\hline
\end{tabular}

Table 5. Factor 5 - External Factors

\begin{tabular}{|r|c|}
\hline BN3 & Slowness in decision making process by owner \\
\hline BN2 & Hydrological and flow factors \\
\hline BN4 & Slowness in supplying materials from distributors \\
\hline BN5 & Accident during construction due to lack of safety measures \\
\hline BN6 & $\begin{array}{c}\text { Geology has many changes such as sliding slopes, flowing } \\
\text { sand, etc }\end{array}$ \\
\hline BN1 & Extreme weather conditions \\
\hline
\end{tabular}

Table 6. Factor 3 - Progress Factors

\begin{tabular}{|r|c|}
\hline QT3 & Delay in progress payments by owner \\
\hline QT5 & $\begin{array}{r}\text { Construction units store materials in contravention of } \\
\text { regulations, causing losses and damage }\end{array}$ \\
\hline QT4 & $\begin{array}{r}\text { Delay to furnish and deliver the site to the contractor by the } \\
\text { owner }\end{array}$ \\
\hline QT2 & Slowness in taking over the completed works by owner \\
\hline QT1 & Delay in supplying design documents by owner \\
\hline
\end{tabular}

Table 7. Factor 4 - Human Factors

\begin{tabular}{|c|c|}
\hline CN3 & Storage of trained labors \\
\hline CN1 & Change in design manager or principal architect \\
\hline CN5 & $\begin{array}{c}\text { Change many sub-contractors or sign contracts with many } \\
\text { constrictors and sub-contractors }\end{array}$ \\
\hline CN4 & Conflicts between owners and other parties \\
\hline CN6 & Conflicts between individuals of construction unit \\
\hline
\end{tabular}

Table 8. Factor 5 - Legal Factors

\begin{tabular}{|c|c|}
\hline PL1 & Local movement are troublesome \\
\hline PL3 & Control sub-contractors through poor contract terms \\
\hline PL2 & Changes in legal documents \\
\hline
\end{tabular}

Table 9. Factor 6 - Technical Factors

\begin{tabular}{|c|c|}
\hline KT7 & Inefficient use of the device \\
\hline KT9 & Unreasonable testing methods \\
\hline KT4 & Low productivity level of labors \\
\hline KT6 & Change in material cost \\
\hline KT3 & Financial constraint of contractors \\
\hline KT2 & Unreasonable schedule \\
\hline KT8 & Low/ unsuitable technology in construction \\
\hline KT5 & Inadequate experience of consultant \\
\hline
\end{tabular}

\subsection{Regression Analysis}

Applying multiple regression methods to test research hypotheses and perform important level measurement of components of the delay with the hypothesis N0: the dependent variables do not have a linear relationship with the indecent variables. $\mathrm{R}^{2}$ values apply great measure about the important scale of relationship between predictor variables, dependent variables and independent variables, with $R^{2}>0.6$. The results of multiple linear regression showed that the coefficient of determination of $\mathrm{R}^{2}$ is 0.736 , meaning that the linear model was built in accordance with data set to $73.6 \%$ (Table 3). The regression equation has the following form:

$$
\mathrm{Y}=\mathrm{a}+\mathrm{b}_{1} \mathrm{X}_{1}+\mathrm{b}_{2} \mathrm{X}_{2}+\ldots \mathrm{B}_{\mathrm{m}} \mathrm{X}_{\mathrm{m}}
$$

Where $\mathrm{Y}$ is dependent variable, $\mathrm{a}$ is constant and is limited at $\mathrm{Y}$ axis; $\mathrm{b}_{1}, \mathrm{~b}_{2}, . . \mathrm{b}_{\mathrm{m}}$ are estimated regression coefficients; $\mathrm{X}_{1}, \mathrm{X}_{2} . ., \mathrm{X}_{\mathrm{m}}$ are predictor values or indecent variables (Table 10).

Table 10. The Result of $\mathrm{R}^{2}$

Model Summary ${ }^{\mathrm{b}}$

\begin{tabular}{|c|c|c|c|c|c|}
\hline Model & $\mathrm{R}$ & R Square & Adjusted R Square & Std. Error of the Estimate & Durbin-Watson \\
\hline 1 & $.736^{\mathrm{a}}$ & .541 & .532 & .379 & 1.862 \\
\hline
\end{tabular}

a. Predictors: (Constant), CN, KT, TK, PL, BN, QT

b. Dependent Variable: Y (TDTC: Schedule Progress) 
Result in Table 11 and Figure 2 is shown that:

$$
\mathrm{Y}=0.919+0.185 \mathrm{~N} 6+0.248 \mathrm{~N} 2+0.079 \mathrm{~N} 1+0.083 \mathrm{~N} 3+0.073 \mathrm{~N} 5+0.18 \mathrm{~N} 4 \text {. }
$$

Table 11. The shorten table of results of regression analysis

\begin{tabular}{|c|c|c|c|c|c|c|c|c|}
\hline \multicolumn{9}{|c|}{ Coefficients $^{\mathrm{a}}$} \\
\hline & \multirow{2}{*}{ Model } & \multicolumn{2}{|c|}{ Unstandardized Coefficients } & \multirow{2}{*}{$\begin{array}{c}\begin{array}{c}\text { Standardized } \\
\text { Coefficients }\end{array} \\
\text { Beta } \\
\end{array}$} & \multirow{2}{*}{$\mathrm{t}$} & \multirow{2}{*}{ Sig. } & \multicolumn{2}{|c|}{ Collinearity Statistics } \\
\hline & & B & Std. Error & & & & Tolerance & VIF \\
\hline \multirow{7}{*}{1} & (Constant) & .919 & .173 & & 5.326 & .000 & & \\
\hline & KT & .185 & .025 & .324 & 7.424 & .000 & .797 & 1.255 \\
\hline & $\mathrm{BN}$ & .248 & .029 & .390 & 8.594 & .000 & .734 & 1.362 \\
\hline & TK & .079 & .030 & .108 & 2.672 & .008 & .925 & 1.082 \\
\hline & QT & .083 & .028 & .136 & 2.969 & .003 & .721 & 1.386 \\
\hline & PL & .073 & .031 & .103 & 2.371 & .018 & .806 & 1.241 \\
\hline & $\mathrm{CN}$ & .180 & .031 & .260 & 5.823 & .000 & .757 & 1.321 \\
\hline
\end{tabular}

a. Dependent Variable: Y (TDTC: Schedule Progress)

\section{Scatterplot}

\section{Dependent Variable: TDTC}

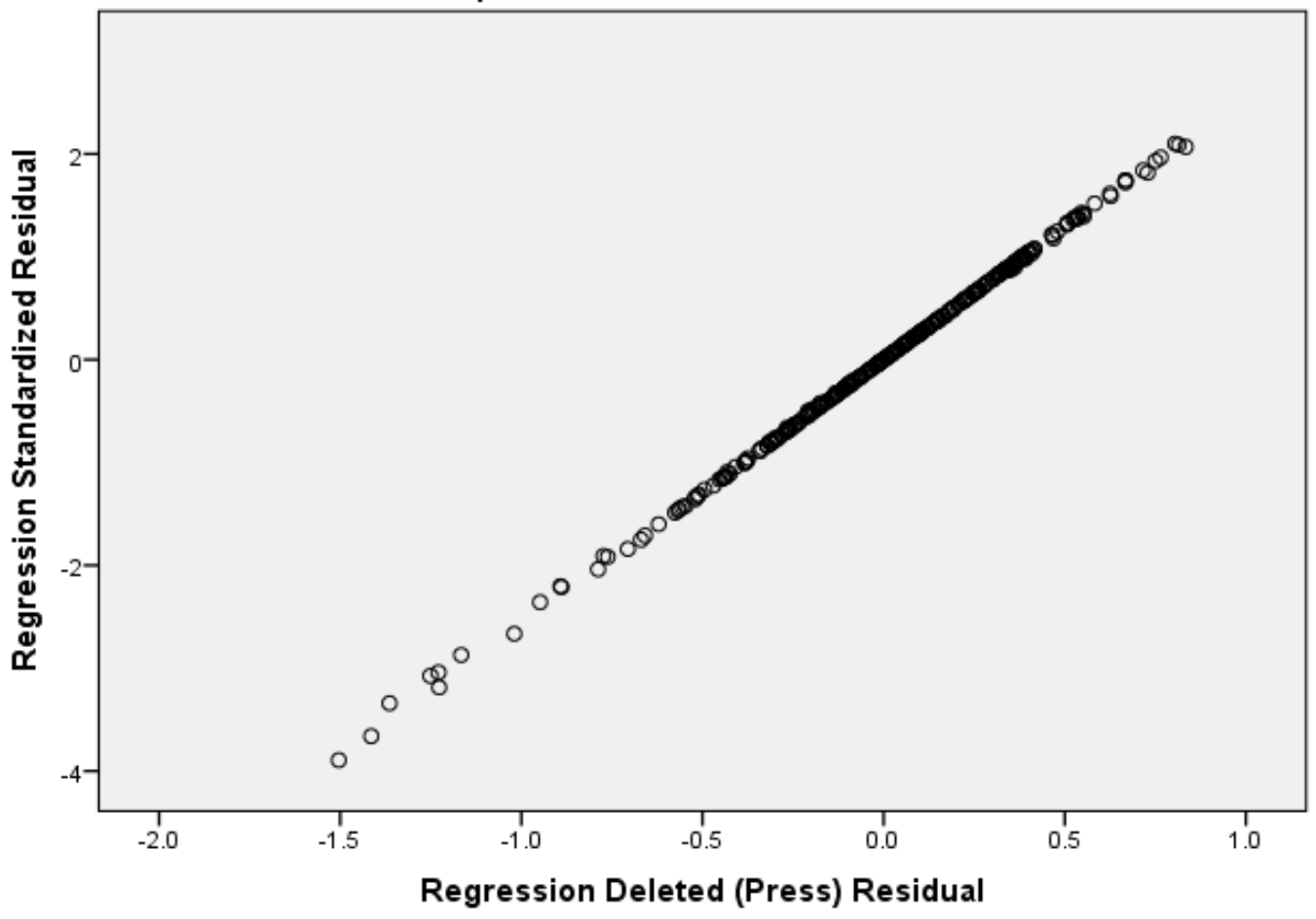

Figure 2. Regression Analysis Plot 


\section{Results and Discussions}

\subsection{Technical Factors}

Through analysis results, technical factors are reflected through eight main factors (Table 12). And inefficient use of the device is a leading factor affecting delay of schedule. In fact, in many construction sites, most contractors take advantage of the available equipment or rented machinery of the units around construction site without calculating, leading to poor coordination between devices and poor performance. Unreasonable testing method is one of the factors effected to delay in construction. Contractors often hire other laboratories to conduct and not regularly on the construction site. Thus, the inspection and testing are not proactive. Technical staff do not know testing methods and depend entirely on laboratory staff, so many methods have to be repeated several times. Low productivity level of labors is due to use untrained labor, lack of proper supervision, or ineffective management skills of on-site supervisors. In case, there is no labors who meet the requirements, recruiting unskilled workers is inevitable. But they need to be trained before taking them to work. Currently, contractors have not done this. Moreover, unreasonable schedule plan is able to delay in construction. It means that engineers are educated too little about making and managing construction progress. At present most of them set up a simple method (Grant), and do not pay attention to uncertain factors and resources. Therefore, it is difficult to manage the progress to meet the requirements. The financial constraint of contractor reflects the contractor's true ability to manage capital. Many contactors distribute capital to several projects leading to shortage of resources. Inexperienced contractors may not be able to solve problems or understand the complexity of the project leading to misunderstandings and confusion.

\subsection{External Factors}

Slowness in decision making process by owner affects directly to delay in construction (Table 13). In the process of implementation, there are many problems that need to be decided by the owners. Therefore, they have to present regularly and promptly to solve the issues as a manager. Hydrological and flow factors greatly affect solutions of leading flow construction, duration and methods of construction. In particular, some earth dams cannot be constructed during the rainy season. Accidents during construction due to lack of safety measures are not only harmful to individuals but also affect labor productivity. Delay in material delivery shows the lack of commitment on the contractor's bidding plan before construction phase of the project. According to Geraldine [17] the lack of understanding of duration leading to the supply of materials may lead to shortage of raw materials, which is one of the important causes of delay in construction. Geology has many changes because most irrigation works are located on weak geology such as rive-bed. Therefore, the construction progress will be affected, when the phenomenon of extruded, flowing sand and high ground water table happens.

Table 12. Factor 6 - Loading Factors and Cronbach Alpha

\begin{tabular}{|c|c|c|c|}
\hline No. & Technical Factors & Loading Factor & Cronbach Alpha \\
\hline KT7 & Inefficient use of the device & 0.870 \\
\hline KT9 & Unreasonable testing methods & 0.868 \\
\hline KT4 & Low productivity level of labors & 0.867 \\
\hline KT6 & Change in material cost & 0.852 \\
\hline KT3 & Financial constraint of contractors & 0.843 \\
\hline KT2 & Unreasonable schedule & 0.830 \\
\hline KT8 & Low/ unsuitable technology in construction & 0.950 \\
\hline KT5 & Inadequate experience of consultant & 0.792 \\
\hline
\end{tabular}

Table 13. Factor 5 - Loading Factors and Cronbach Alpha

\begin{tabular}{|c|c|c|c|}
\hline No. & External Factors & Loading Factor & Cronbach Alpha \\
\hline BN3 & Slowness in decision making process by owner & 0.839 & \\
\hline BN2 & Hydrological and flow factors & 0.821 \\
\hline BN4 & Slowness in supplying materials from distributors & 0.788 & \multirow{0}{0}{0.906} \\
\hline BN5 & Accident during construction due to lack of safety measures & 0.785 \\
\hline BN6 & Geology has many changes such as sliding slopes, flowing sand, etc & 0.772 \\
\hline BN1 & Extreme weather conditions & 0.756 & \\
\hline
\end{tabular}




\subsection{Design Factors}

Unclear explanation of the ambiguous details in design file misleads field engineer and may lead to wrong construction compared to design ideals, which causes long construction duration. Changing design during construction is often due to differences between the actual and original design; due to change the scope of work compared to original investment policy. It means that the construction time is extended because of waiting adjusted designs along with approval decisions. Moreover, some of design details are wrong, the constrictor must rework due to errors during construction and it becomes one of the reason to delay in construction. In construction, geological survey is particularly important when designing construction works. Therefore, poor surveys, incorrect geological assessments are not only wasting project budget but also slowing the construction progress (Table 14).

\subsection{Progress Factors}

Clearance is the most pressing issue in most projects in Vietnam not only for irrigation and hydropower projects (Table 15). There are many projects showing that the time construction may exceed 2 to 3 years because of failing to hand over the site. According to the collected answer sheet, delay in progress payments by owner is also a factor that makes the contractors unable to arrange construction capital in time, then, it increases construction duration. From many viewpoints of respondents, the contractor who holds the materials improperly in the warehouse causes loss and waste substance, affecting the contractor's financial resources is also a major factor of delay.

\subsection{Human Factors}

Human factor is considered the subject in the relationship between participants in construction process (Table 16). In particular, lack of professional technical staff is the current popular situation in Vietnam. Contractors tend to recruit technicians in the short term because of profit problem, leading to no professional and high-tech expertise. Therefore, the time to implement work meets a lot of difficulty. In many projects, there will be the appearance of sub-contractors to perform some parts of contract. However, changing and signing contract with many sub-contractors, other contractors reflect the capacity of main contractor. And the problem of inefficient controlling sub-contractors leads to chaos on construction site, overlap and shortage in implementation process. In additions, conflicts between joint-ownership of the project, or between internal contractors also increase construction duration.

Table 14. Factor 1 - Loading Factors and Cronbach Alpha

\begin{tabular}{|c|c|c|c|}
\hline No. & Design Factors & Loading Factor & Cronbach Alpha \\
\hline TK2 & Unclear and inadequate details in drawings & 0.822 & \\
\hline TK4 & Change the design during construction time & 0.796 \\
\hline TK6 & Misunderstanding of owner's requirements by design engineer & 0.784 & 0.810 \\
\hline TK3 & Insufficient data collection and survey before design & 0.731 \\
\hline TK1 & Increase the work compared to the original investment policy & 0.730 & \\
\hline
\end{tabular}

Table 15. Factor 3 - Loading Factors and Cronbach Alpha

\begin{tabular}{|c|c|c|c|}
\hline No. & Progress Factors & Loading Factor & Cronbach Alpha \\
\hline QT3 & Delay in progress payments by owner & 0.828 & \\
\hline QT5 & $\begin{array}{c}\text { Construction units store materials in contravention of regulations, causing losses and } \\
\text { damage }\end{array}$ & 0.824 \\
\hline QT4 & Delay to furnish and deliver the site to the contractor by the owner & 0.798 & 0.884 \\
\hline QT2 & Slowness in taking over the completed works by owner & 0.788 \\
\hline QT1 & Delay in supplying design documents by owner & 0.691 & \\
\hline
\end{tabular}

Table 16. Factor 4 - Loading Factors and Cronbach Alpha

\begin{tabular}{|c|c|c|c|}
\hline No. & Human Factors & Loading Factor & Cronbach Alpha \\
\hline CN3 & Storage of trained labors & 0.854 & \\
\hline CN1 & Change in design manager or principal architect & 0.808 \\
\hline CN5 & Change many sub-contractors or sign contracts with many constrictors and sub-contractors & 0.802 & 0.894 \\
\hline CN4 & Conflicts between owners and other parties & 0.789 \\
\hline CN6 & Conflicts between individuals of construction unit & 0.774 & \\
\hline
\end{tabular}


Table 17. Factor 5 - Loading Factors and Cronbach Alpha

\begin{tabular}{|c|c|c|c|}
\hline No. & Legal Factors & Loading Factor & Cronbach Alpha \\
\hline PL1 & Local movement are troublesome & 0.857 & \\
\hline PL3 & Control sub-contractors through poor contract terms & 0.841 & 0.847 \\
\hline PL2 & Changes in legal documents & 0.799 & \\
\hline
\end{tabular}

\subsection{Legal Factors}

The issue of administrative procedure reform has been concerned from central to local level in Vietnam. But most of the procedures are still complicated, taking a lot of time with many stages. Besides, corruption and harassment in some places also prolong project's duration. Although, it is considered one of the least important causes of delay, regular changes in legal documents and circulars are also affecting the implementation process. Besides, controlling sub-contractors with poor contract terms is a common situation today (Table 17).

\section{Conclusions and Recommendations}

This research shows that the most influential factor in construction delays is technical factors: unreasonable construction schedule, organize construction method, etc. This happens mostly on the existing irrigation and hydropower construction project, especially construction schedule and measures to coordinate motorcycles and construction equipment on sites. It shows that training engineers in professional construction progress is essential. This research also finds out main external factors influencing project delays are: Hydrological and flow conditions, slowness in decision making process by owner. This explains for many projects that have been done through in practices such as Cua Dat hydropower in Thanh Hoa province, Ta Trach in Thua Thien Hue province, Phu Vinh in Quang Binh province. Besides, human factors are also highly influential, especially lack of professional human resources, changes in sub-contractors. This clearly reflects the current situation in most irrigation and hydropower projects in Vietnam, that is, contractors do not have professional technical staff so they often hire untrained labors who usually live near construction site. Other factors such as changes in design file, legal environment, delay in progress payment and supplying documents by owner less affect schedule. Although there is clear understanding of these key factors among the subjects involved in construction, but the real efforts to minimize these risks have not yet really focused in Vietnam. Traditionally, the approach of establishing and managing construction progress is still quite simple, so it is necessary to apply systematically. In the world, there have been many researches and solutions on construction and management of construction progress, but in Vietnam still use simple methods and based largely on construction norms set by the ministry. Therefore, through this research, author has analyzed and clearly assessed the current situation of factors affecting delay in construction of irrigation and hydropower in Vietnam especially dam works. Hence, there are orientations for developing an appropriate plan and management method of construction in Vietnam, including uncertainty factors and reliability theory. These analyses contribute significantly to the construction of irrigation and hydropower in Vietnam.

Although this research tries to come up with findings that have contributed significantly to the construction of irrigation and hydropower in Vietnam, it has some limitations. First, the respondents are not evenly distributed among the roles, which may have caused some bias in the assessment. Secondly, this research does not have detailed analysis of effect of each factor on construction delays, thereby giving a method of setting up the construction schedule according to the theory of reliability. This will be the next research of the authors.

\section{REFERENCES}

[1] General Statistics Office of Vietnam. General Statistics Report 2020 of Vietnam. Statistics Publishing House of Vietnam, 2021.

[2] Hue N.H., Son N.V, "Evaluate the level of Effect of Delay Schedule Factors on Irrigation and Hydropower Works in Vietnam. Journal of Water Resources and Environmental Engineering, Vol. 68/12-2019, 2019, pp. 93-100.

[3] Vietnam National Committee on Large Dams (VNCOLD), "Carry out the progress of the implementation of irrigation projects invested with Government bonds", Internal Research on Large Dams Management Systems, 2019, 257 pages.

[4] Luan P.H, "The causes affecting delays of construction projects in Vietnam”, Journal of Construction of Vietnam, Vol 2006(7), 2006, pp. 16-28.

[5] Lan T.H, "Research to determine the progress of construction works taking into account uncertainty, applied to a number of projects in Vietnam”, Master Thesis of National University of Civil Engineering of Vietnam, 2012, 120 pages.

[6] Dung L.A, "Optimize the progress of construction works", Doctoral Dissertation, Hanoi Architectural University, 
2004, 150 pages.

[7] Chan D.W, "A comparative study of causes of time overruns in Hong Kong construction projects", International Journal of Project Management, Vol. 15, 1997, pp. 55-63.

[8] Al-Ghafly M.A, "Delays in construction of public utility projects in Saudi Arabia”, International Journal of Project Management, Vol. 17, 1999, pp. 101-106.

[9] Al-Momani A.H, “Construction delay: a quantitative analysis”, International Journal of Project Management, Vol. 18, 2000, pp. 51-59.

[10] Iyer K.C, and Jha K.N, "Factors affecting cost performance: evidence from Indian construction projects”, International Journal of Project Management, Vol. 23, 2005, pp. 43-56.

[11] Sadi A.A., and Sadiq A, "Causes of delay in large construction projects", International Journal of Project Management Vol. 24, 2006, pp. 349-357.

[12] Long L.H., Lee Y.D., Lee Y.Y, "Delay and cost overruns in Vietnam large construction project: A comparison with other selected countries," KSCE Journal of Civil Engineering, 2008, pp. 367-377.

[13] El-Razek A.M.E., Bassioni H.A., Mobarak A.M, "Causes of delay in building construction projects in Egypt", Journal of Construction Engineering Management, Vol. 134, 2008, pp. 89-102.
[14] Al-Kharashi A., Skitmore, M, "Causes of delays in Saudi Arabian public sector construction projects”, Journal of Construction Management and Economics, Vol. 54, 2009, pp. 65-74.

[15] Hao C.T., Tam N.T.M, "Factors affecting the cost fluctuation of construction projects", Master Thesis, Ho Chi Minh City University of Polytechnics, 2009, 120 pages.

[16] Olawale Y.A., Sun M, "Cost and time control of construction projects: inhibiting factors and mitigating measures in practice”, Construction Management and Economics, Vol. 28, 2010, pp. 109-134.

[17] Geraldine J.K, "Causes and effects of delays and disruptions in construction projects in Tanzania", Australasian Journal of Construction Economics and Building, Conference Series, 2012, pp. 52-59.

[18] Anh T.T, "Factors causing delay in transport projects using state budget capital in the southern provinces", Economic-technical journal, Vol. 36(12-2014), 2014, pp. 564-572.

[19] Lam V.Q, "Factors causing delay progress and exceeding estimates of public investment projects in Vietnam", Journal of Development and Integration, Vol. 23, 2015, pp. 24-31.

[20] Ramanathan J.K, "Construction Delays Causing Risks on Time and Cost - a Critical Review”, Australasian Journal of Construction Economics and Building, Vol. 12, 2018, pp. 37-57. 